\title{
Capacitance-Power-Hysteresis Trilemma in Nanoporous Supercapacitors
}

\author{
Alpha A. Lee, ${ }^{1,2}$ Dominic Vella, ${ }^{2}$ Alain Goriely, ${ }^{2}$ and Svyatoslav Kondrat ${ }^{3,4}$ \\ ${ }^{1}$ School of Engineering and Applied Sciences, Harvard University, Cambridge, Massachusetts 02138, USA \\ ${ }^{2}$ Mathematical Institute, Andrew Wiles Building, University of Oxford, Woodstock Road, \\ Oxford OX2 6GG, United Kingdom \\ ${ }^{3}$ IBG-1: Biotechnology, Forschungszentrum Jülich, 52425Jülich, Germany \\ ${ }^{4}$ Department of Chemistry, Faculty of Natural Sciences, Imperial College London, \\ SW7 2AZ, United Kingdom
}

(Received 23 June 2015; revised manuscript received 13 March 2016; published 14 June 2016)

\begin{abstract}
Nanoporous supercapacitors are an important player in the field of energy storage that fill the gap between dielectric capacitors and batteries. The key challenge in the development of supercapacitors is the perceived trade-off between capacitance and power delivery. Current efforts to boost the capacitance of nanoporous supercapacitors focus on reducing the pore size so that they can only accommodate a single layer of ions. However, this tight packing compromises the charging dynamics and hence power density. We show via an analytical theory and Monte Carlo simulations that charging is sensitively dependent on the affinity of ions to the pores, and that high capacitances can be obtained for ionophobic pores of widths significantly larger than the ion diameter. Our theory also predicts that charging can be hysteretic with a significant energy loss per cycle for intermediate ionophilicities. We use these observations to explore the parameter regimes in which a capacitance-power-hysteresis trilemma may be avoided.
\end{abstract}

DOI: 10.1103/PhysRevX.6.021034

\section{INTRODUCTION}

The physics of charge storage at the nanoscale has received significant attention in recent years because of its relevance for efficient energy storage and the development of novel green technologies [1-3]. In particular, extensive effort has been channeled into studying electrical double-layer capacitors (also called supercapacitors) in which energy is stored at the electrode-electrolyte interface. Their importance for energy storage has stimulated the development of novel techniques for fabrication of conducting nanoporous materials $[3,4]$. For instance, high-temperature chlorination of carbides produces carbon electrodes with a network of slit and/or cylindrical nanopores with narrow pore-size distribution about a well-controlled average pore size [5]. There is also an emerging class of graphene-based electrodes consisting of aligned slit nanopores with pore sizes comparable to the ion diameter $[6,7]$. Additionally, it is possible to manipulate the ion-pore interactions by functionalizing carbons. For instance, preparing carbon nanofibers in the presence of potassium hydroxide changes the surface functionality and increases the ion-pore attraction [8].

Published by the American Physical Society under the terms of the Creative Commons Attribution 3.0 License. Further distribution of this work must maintain attribution to the author(s) and the published article's title, journal citation, and DOI.
Subject Areas: Energy Research, Materials Science, Soft Matter
Nanoporous supercapacitors benefit from a high surfaceto-volume ratio of these materials with an increase in the volumetric capacitance observed as the surface area of the electrode increases [9]. However, pioneering experiments [10-13] have shown that a drastic increase in surfacespecific capacitance is achieved when the average pore size approaches the ion diameter. Using a model of a single metallic slit-shaped nanopore (cf. Fig. 1), this "anomalous" increase of capacitance has been explained by the emergence of a "superionic state" in which the interionic interactions become exponentially screened. Decreasing the pore size promotes screening, which decreases the energy penalty for packing like charges and unbinding ion pairs, purportedly leading to an increase in the capacitance [14]. This reasoning also applies to nonperfect metals [15-18]. In particular, recent quantum density-functional calculations have shown that the ion-ion interactions are



FIG. 1. Schematic drawing of the model porous electrode under consideration: Ions of diameter $d$ are confined between two metallic surfaces separated by a distance $L$. A potential $V$ (relative to the bath) is applied to the surfaces. 
exponentially screened in carbon nanotubes [16,17], and it is reasonable to expect a similar behavior for other types of confinement, including slit pores.

This effect of metallic screening has been observed in molecular-dynamics simulations that use more elaborate models to account for complex pore geometries [19,20] and realistic ions [21-23]. If metallic screening is the sole driver of increased capacitance, the capacitance can only be optimized when the pore size equals the ion size. However, such a close-fitting pore is detrimental to the charging dynamics [24] because of the reduced effective diffusivity [25-27] and because the kinetic barrier to pore entry is large. Increased capacitance therefore appears to come at the cost of prolonged charging. This leads to a dilemma in the design of supercapacitors-should the design be chosen to optimize capacitance or power? Alternatively, one might naturally ask the following question: Can the capacitance be maximized away from sterically close-fitting pores?

It is known that charging of flat electrodes may show a hysteresis [28-31]; i.e., the differential capacitance depends on the initial voltage and the direction of scan $[28,32]$. This effect is connected with the existence of two or more metastable states of an ionic liquid at the electrode surface [31]. In nanoconfinement, there is evidence from a mean-field study [14], Monte Carlo [33], and molecular dynamics [34,35] simulations that charging of slit nanopores can proceed via a voltage-induced discontinuous transition that is manifested by an abrupt change in the ion density. Such discontinuous transitions can be detrimental to the operation of supercapacitors because of hysteretic energy losses when the charging and discharging routes follow different metastable branches. It is thus important to know whether (and when) charging is hysteretic and how hysteresis might be avoided altogether. This problem adds another dimension to the dilemma already mentioned.

To answer these questions, we combine a mean-field theory with Monte Carlo simulations for a model-slit nanopore (Fig. 1). We consider monovalent ions and a single slit-shaped metallic nanopore. The pore entrance and closing are ignored, and charging is modeled by applying a nonzero potential to the pore walls. Similar models have previously been used to study charge storage $[14,23,33,36-$ 40] and the dynamics of charging [25,27] of nanoporous supercapacitors. Here, we focus specifically on pores whose sizes are comparable with the ion diameter. In this limit, the system is quasi two-dimensional, and we can assume that ions are located on the symmetry plane of the pore. These assumptions allow us to develop a mean-field theory in two dimensions, whereby, improving on a model developed previously [14], the entropy of out-of-plane packing of ions (for $L>d$ ) is taken into account by introducing an effective pore-width-dependent ion diameter $d^{*} \leq d$ (see Appendix A 2; we neglect the out-of-plane effects of electrostatic interactions as they are subdominant). We supplement and compare our mean-field results with grand canonical Monte Carlo simulations of the same system in three dimensions (for simulation details, see Appendix B).

The model employed here ignores the chemical details of ions and the complex structures of nanoporous electrodes. With these deliberate simplifications, we aspire to reveal and understand the essential physics at work, without the complexity of real supercapacitors. In particular, our study reveals that, contrary to the long-standing paradigm, a maximal capacitance can actually be achieved when pores are appreciably wider than the ion diameter. In addition, we show that there is, in general, charging hysteresis with significant energy loss per cycle. However, this hysteresis can be evaded by carefully tuning the ion-pore interaction energy. Our study therefore reveals generic features of such systems that we believe should apply more generally and hence may provide a framework within which to design optimal nanoporous supercapacitors and avoid the capacitance-power-hysteresis trilemma discussed above.

\section{IONOPHOBICITY OF PORES}

Our primary interest is to determine how the affinity of ions towards pores affects charging. In our model, this affinity is controlled by the electrochemical potential,

$$
h_{ \pm}= \pm e V+\delta E_{\text {self }}+\delta E_{ \pm},
$$

where $V$ is the applied voltage, $e$ the elementary charge, $\delta E_{\text {self }}$ the ions' self-energy [see Eq. (A9) in Appendix A 3], and $\delta E_{ \pm}$the resolvation energy [14]. The resolvation energy is the energy of transferring an ion from the bulk to the pore. We assume $\delta E_{+}=\delta E_{-}=\delta E$ for simplicity.

At zero applied voltage, $h_{ \pm}=h_{0}=\delta E_{\text {self }}+\delta E$, and the sign of $h_{0}$ determines whether the ion-pore interactions are favorable. For pores with $h_{0}>0$ (unfavorable ion-pore interaction), the occupancy of the pore at zero applied voltage is expected to be low; we therefore refer to such pores as ionophobic. For large positive $h_{0}$, the pore will be (almost) completely free of ions at zero applied voltage; we therefore refer to such pores as strongly ionophobic. Conversely, pores with $h_{0}<0$ will be termed ionophilic, and a large negative $h_{0}$ will correspond to strongly ionophilic pores, which are nearly fully occupied by ions at no applied voltage. Here, $h_{0}=0$ marks the crossover between ionophilic and ionophobic behaviors, which therefore occurs at a resolvation energy $\delta E=\delta E_{\text {crossover }}=$ $-\delta E_{\text {self }}=l_{B} \ln (2) / L$ (in units of $k_{B} T$ ), where $l_{B}$ is the Bjerrum length (see Appendix A 3 for an estimate of $\left.\delta E_{\text {self }}\right)$. For instance, for a typical Bjerrum length of $25 \mathrm{~nm}$ and a 0.7 -nm-wide pore, we obtain $\delta E_{\text {crossover }} \approx$ $25 k_{B} T$, while for a 0.6 -nm-wide pore we have $\delta E_{\text {crossover }} \approx$ $29 k_{B} T$ (vertical lines in Fig. 2).

To obtain an estimate for the resolvation energy, we decompose $\delta E$ into $\delta E \approx \delta E_{\text {desolv }}+\delta E_{\text {nonele }}$, where $\delta E_{\text {desolv }}$ is the desolvation energy of the bulk ionic liquid 


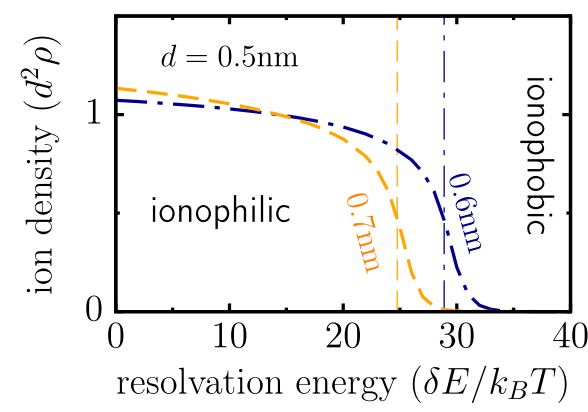

FIG. 2. An ion's resolvation energy, $\delta E$, determines the ion density (per surface area) inside nanopores at no applied voltage. At large $\delta E$, ions prefer to stay outside the pores, and the pores are (nearly) free of ions; we term such pores ionophobic. In the opposite case of small or negative $\delta E$, the pores are occupied by ions, and we call them ionophilic. The crossover between ionophilic and ionophobic occurs when $\delta E_{\text {crossover }}=$ $l_{B} \ln (2) / L$, where $l_{B}$ is the Bjerrum length and $L$ the pore width (see text); $\delta E_{\text {crossover }}$ for two pore sizes $(L=0.6 \mathrm{~nm}$ and $L=0.7 \mathrm{~nm}$ ) are shown by thin vertical lines. Pores with $\delta E$ close to $\delta E_{\text {crossover }}$ have moderate ion densities at zero applied voltages, and we term them weakly ionophilic/ionophobic. The ion diameter is $d=0.5 \mathrm{~nm}$, and the Bjerrum length is $l_{B}=25 \mathrm{~nm}$. This plot has been obtained by using the mean-field theory (see Appendix A).

(transferring one solvated ion from the bulk liquid to the vapor state), and $\delta E_{\text {nonele }}$ is the ion-pore nonelectrostatic interaction (ion-pore electrostatic interactions are accounted for in $\delta E_{\text {self }}$ ). A combination of quantummechanical density functional calculations and molecular dynamics simulations [41] suggests $\delta E_{\text {desolv }} \approx 65-$ $110 k_{B} T$ per ion [42]. The main source of nonelectrostatic interactions is the van der Waals attraction, of magnitude $\delta E_{\mathrm{vdW}} \approx-70 k_{B} T$ [43]. We thus find that, roughly, $-5 \lesssim$ $\delta E /\left(k_{B} T\right) \lesssim 45$ (note that the crossover between ionophobic and ionophilic pores, $\delta E_{\text {crossover }}$, lies in this range). We stress that this range is not exhaustive, as other physical effects (such as specific surface chemistry of the pore or the presence of solvent) have not been taken into account in this analysis. In particular, recent experimental $[44,45]$ and theoretical [46] studies suggest that ionophobicity can be effectively controlled by changing the solvent concentration. In any case, we emphasize that the definitive metric of ionophilicity or ionophobicity is the occupancy of the pore at zero applied voltage, as shown in Fig. 2.

\section{SEARCHING FOR MAXIMAL CAPACITANCE}

The key quantity characterizing the low voltage capacitance response of a supercapacitor is the differential capacitance at zero voltage,

$$
C_{D}(0)=\left.\frac{\mathrm{d} Q}{\mathrm{~d} V}\right|_{V=0},
$$

where $Q$ is the charge stored in a pore, and $V$ is the applied potential. In the following discussion, we compute $Q(V)$ by minimizing a mean-field free-energy function or directly from Monte Carlo simulations (as discussed in Appendix B). Clearly, $Q(0)=0$ by electroneutrality, while $C_{D}(0)$ describes the response of the system to an increment in the applied voltage and is, in general, nonzero.

The mean-field approximation for the Helmholtz free energy is given by

$$
\beta F=U_{\mathrm{el}}\left(\rho_{+}, \rho_{-}\right)-S\left(\rho_{+}, \rho_{-}\right)+\sum_{\alpha= \pm} h_{\alpha} \rho_{\alpha},
$$

where $\beta=\left(k_{B} T\right)^{-1}$ (with $k_{B}$ being the Boltzmann constant and $T$ temperature) and $\rho_{ \pm}$the two-dimensional density of \pm ions. Here, $k_{B} T U_{\mathrm{el}}$ is the contribution to the free energy due to electrostatic interactions and $k_{B} T S$ is the excluded volume entropic contribution; we assume that the density of ions in the slit pore is homogeneous (see Appendix A for expressions of $U_{\mathrm{el}}$ and $S$ ). To obtain $Q(V)$ and hence $C_{D}(0)$, we minimize $F$ over $\rho_{ \pm}$subject to fixed $V$, noting
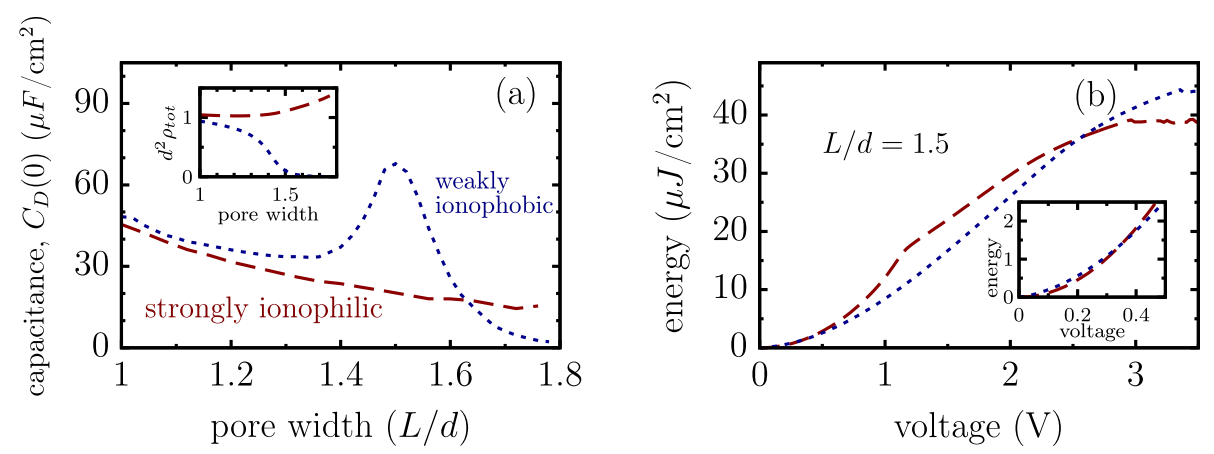

FIG. 3. (a) Differential capacitance at zero voltage, $C_{D}(0)$, as a function of pore width and (b) the stored energy as a function of voltage (for $L=0.75 \mathrm{~nm}$ ) from the mean-field theory, plotted for weakly ionophobic $\left(\delta E=25 k_{B} T\right)$ and strongly ionophilic $\left(\delta E=10 k_{B} T\right)$ pores, as indicated by the line styles on the plots. The ion diameter is $0.5 \mathrm{~nm}$. The inset in (a) shows how the total ion density at zero voltage depends on pore width. The inset in (b) highlights the stored energy at low voltages. 
that the charge per unit area is $Q=e\left(\rho_{+}-\rho_{-}\right)$, where $e$ is the elementary charge.

It is possible that the free energy has two minima, one of which can be metastable (i.e. it is a local but not global minimum), and charging and discharging may follow different metastable branches. This possibility will be discussed in Sec. IV. We first focus on the parameter regime where the free-energy minimum is unique.

For strongly ionophilic pores, we find that the capacitance is maximal for the smallest pore possible, in agreement with the conventional view that the capacitance increases monotonically as the pore size decreases [10-13] [dashed line in Fig. 3(a)]. Surprisingly, however, we find that for weakly ionophobic pores, the differential capacitance has a global maximum when the pore width is significantly larger than the ion diameter (though still smaller than $2 d$ ). This behavior is due to two competing effects: On the one hand, the loss of ion-image interactions and an increase in electrostatic interactions hinders ions from entering the pore. On the other hand, the same factors also render the pore less populated at zero voltage [inset in Fig. 3(a)]. As the width varies, a peak is achieved when the decrease in the total density frees up enough space in the pore, so that counter-ions insertion becomes entropically favorable. In fact, for narrow and weakly ionophilic pores, the charging at low voltages is dominated by swapping coions for counterions and expelling coions; for wider pores, it is the counterion insertion that drives charging.

This charging behavior is in contrast to strongly ionophilic pores, where total ion density increases with increasing pore width because the out-of-plane degrees of freedom allow ions to pack more efficiently. Therefore, both entropy and ion-ion as well as ion-image interactions work against charging as the pore size $(L)$ increases, and so the capacitance decreases monotonically for increasing $L$. The case of strongly ionophobic pores is not considered here-charging commences only when ions can overcome the ionophobicity barrier $(e V \approx \delta E)$, and hence the capacitance at zero voltage is low or vanishing.

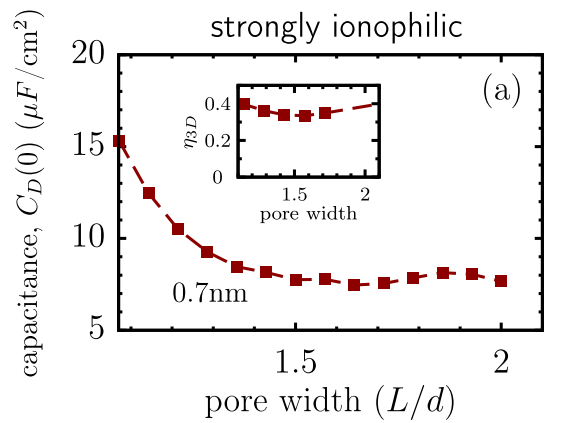

Figure 3(b) shows the energy per surface area stored for each of the two pores. For low applied voltages, the stored energy is slightly higher for the weakly ionophobic pore [inset in Fig. 3(b)] because it has a higher low-voltage capacitance. For intermediate voltages, however, capacitance is higher for ionophilic pores [cf. Fig. 6(a)], and weak ionophobicity reduces the energy storage in this voltage range. For sufficiently high voltages, ionophilic pores eventually saturate, while ionophobic pores continue to charge; this ultimately leads to higher energies stored by ionophobic pores $[47,48]$.

Monte Carlo simulations confirm the predicted trends of the mean-field model. Strongly ionophilic pores show a monotonic decrease of capacitance with increasing pore size [Fig. 4(a)], while weak ionophobicity produces a local maximum with capacitances comparable to, or even higher than, the capacitance at $L \approx d$ [Fig. 4(b)]. The total ion packing fraction is almost constant with increasing slit width for strongly ionophilic pores [inset of Fig. 4(a)], whereas for ionophobic pores, it decreases with increasing slit width [inset of Fig. 4(b)], agreeing qualitatively with the mean-field model [inset of Fig. 3(a)]. We note that a slightly smaller $\delta E$ in mean-field theory is sufficient to achieve the same ion occupancy at zero voltage, hence ionophobicity, as in the Monte Carlo simulations. This discrepancy is likely due to the fact that our mean-field theory does not account for the change in ion-image interactions due to ions positioning themselves off the central symmetry plane of the pore (though this effect does not change the qualitative predictions of the mean-field model).

The presence of a local maximum is distinct from the oscillatory behavior of capacitance as a function of pore width [39,49,50] and solvent polarity [40] observed in systems with wider pores. For weakly ionophobic pores, a maximum occurs because of entropic effects, while for systems with larger pores, it is the overlapping double layers and highly polar solvent, respectively, that cause the nonmonotonicity in the capacitance [notice a second maximum in Fig. 4(a), with a similar origin but with a much smaller amplitude].

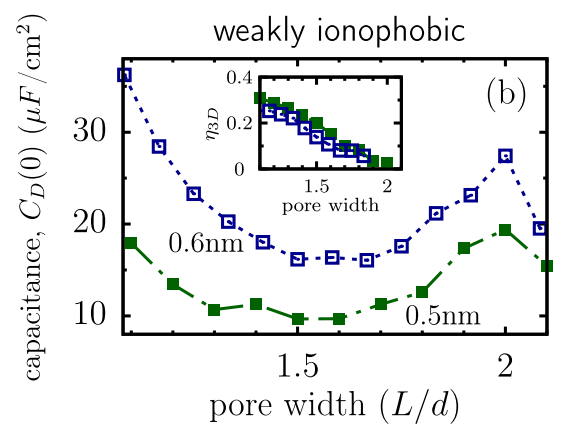

FIG. 4. The calculated dependence of the differential capacitance, $C_{D}(0)$, as the pore width varies. The results of Monte Carlo simulations are shown for a strongly ionophilic pore $\left(\delta E=-2.5 k_{B} T\right)$ and for weakly ionophobic pores $\left(\delta E=38.5 k_{B} T\right.$ for $d=0.5 \mathrm{~nm}$ and $\delta E=33.2 k_{B} T$ for $\left.d=0.6 \mathrm{~nm}\right)$. The insets show the $3 \mathrm{D}$ packing fraction $\eta_{3 \mathrm{D}}=(\pi / 6) \rho d^{3}$ as a function of pore width. 

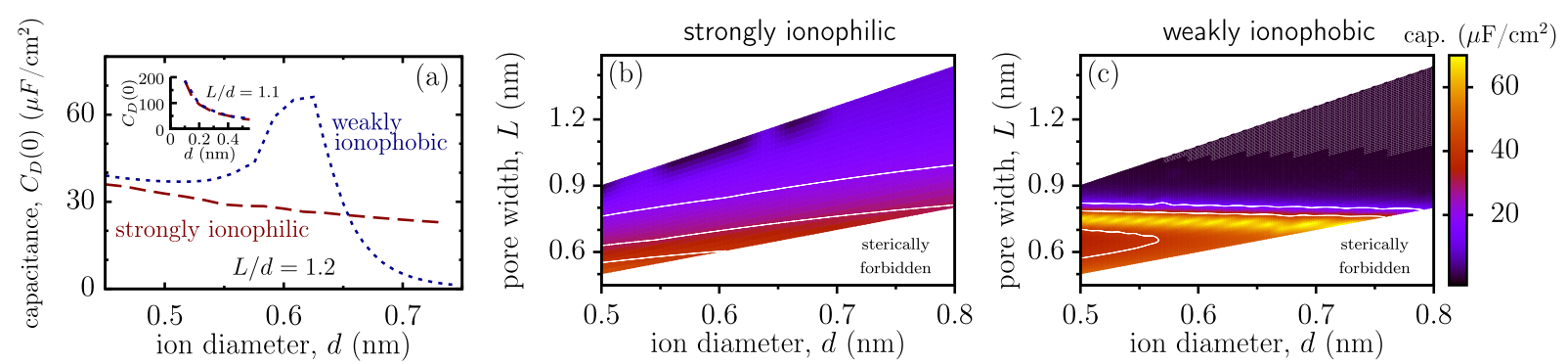

FIG. 5. (a) Capacitance at zero voltage, $C_{D}(0)$, as a function of ion diameter $\left(d=d_{ \pm}\right)$for weakly ionophobic $\left(\delta E=25 k_{B} T\right)$ and strongly ionophilic $\left(\delta E=10 k_{B} T\right)$ pores calculated using mean-field theory (MFT). The ratio between the pore width and the ion diameter is fixed to $L / d=1.2$. The inset shows $C_{D}(0)$ for small ions with $d \lesssim 0.5 \mathrm{~nm}$ and $L / d=1.1$. For such ultra narrow pores, $C_{D}(0)$ is practically independent of ionophobicity (for the values of $\delta E$ used in this plot). This is because the image forces [see Eq. (A9) in Appendix A 3] overcompensate $\delta E$ for slit widths $L \lesssim 0.55$, making the pores completely filled with ions. (b,c) Capacitance map for strongly ionophilic and weakly ionophobic nanopores and room-temperature ionic liquids (with $d \gtrsim 0.5 \mathrm{~nm}$ ) in the plane of ion diameter $(d)$ and the pore width $(L)$ calculated using MFT. This figure suggests a "two-step optimization strategy," in which an ionophobicity-dependent optimal pair $(d, L)$ exists that maximizes the differential capacitance.

Another important aspect of capacitance optimization is how to choose the ion size $(d)$ and slit width $(L)$. Intuitively, one might expect that it is the ratio between the two that affects the capacitance. However, Fig. 5(a) shows that for weakly ionophobic pores, a peak in the capacitance emerges as the ion diameter increases, while the ratio between the slit width and the ion diameter, $L / d$, is kept fixed. This peak occurs because the ion self-energy and the metallic screening decrease (i.e., electrostatic interactions become stronger) when increasing the ion diameter at constant $L / d$ [see Eqs. (A3) and (A9) in Appendix A]. This effect makes the pore less populated for weak ionophobicities, and thus adsorption of new counterions becomes entropically more favorable, giving rise to a local maximum at intermediate ion sizes. For strongly ionophilic pores, the ion density is close to maximal, and charging proceeds mainly via swapping coions for counterions. Therefore, the capacitance increases monotonically with decreasing ion diameter [dashed curve in Fig. 5(a)].

From these results, we see that the highest possible capacitance may be obtained by optimizing both the pore width and the ion diameter [see Figs. 5(b) and 5(c)]. Crucially, the position of this optimum in parameter space, and its properties, depend on the pore's ionophilicity. For strongly ionophilic pores, the pores are completely filled with ions, and a maximal capacitance is achieved for small ions and tight pores; i.e., $L_{\mathrm{opt}} \approx d$ for any $d$, and the capacitance increases as $d$ decreases [Figs. 5(a) and 5(b)]. Remarkably, a strong increase of capacitance is obtained for pores below $0.5 \mathrm{~nm}$, reaching values as high as $200 \mu F / \mathrm{cm}^{2}$ [see the inset in Fig. 5(a)]. This result suggests that such ultranarrow pores combined with inorganic electrolytes with small ions may be beneficial for the charge and energy storage.

For weak ionophobicities, there is an optimal slit width $L_{\mathrm{opt}} \approx 0.74 \mathrm{~nm}$ that depends only weakly on the ion diameter $d$, for $d \gtrsim 0.5 \mathrm{~nm}$ [Fig. 5(c)]. As discussed, this is connected with emptying (half-filled) pores, which maximizes the capacitance, i.e., $L_{\text {opt }} \approx L_{\text {empty }}$ [see Figs. 3(a) and 4(b)]. Since the image-force interactions are ion-size independent [see Eq. (A9) in Appendix A 3], we roughly estimate that the pore is emptied when $L \gtrsim L_{\text {empty }} \approx l_{B} \ln (2) / \delta E$ [51]. For the parameters of Fig. 5(c), $l_{B}=25 \mathrm{~nm}$ and $\delta E=25$ (in units of $k_{B} T$ ), we obtain $L_{\text {empty }} \approx 0.7 \mathrm{~nm}$. This value is slightly modified by the ion-size-dependent entropic and screened electrostatic interactions, which additionally bring a weak $L_{\text {opt }}(d)$ dependence.

To conclude this section, the fact that the capacitance reaches a maximum for pores that are significantly wider than the ion diameter may have an important impact on optimizing supercapacitors. It has previously been assumed that there is necessarily a trade-off between having large capacitance (narrow pores) and fast charging (wide pores) [25]. Charging is slower for narrower pores because the collective (or effective) diffusion coefficient $D_{\text {eff }}$, which determines the rate of charging, decreases with decreasing pore width, $L$ [25-27]; for instance, $D_{\text {eff }}$ is almost doubled when $L$ increases from $0.7 \mathrm{~nm}$ to $0.9 \mathrm{~nm}$ [25]. Our analysis therefore provides the key insight that charging kinetics and capacitance can be simultaneously optimized by tuning the ionophilicity of the pore.

\section{HYSTERETIC CHARGING}

Next, we study the charging hysteresis for nonzero applied voltages. Figure 6 shows that charging can proceed via a first-order discontinuous phase transition at intermediate ionophilicities, or via a continuous process beyond the critical endpoints.

For strongly ionophobic pores [with resolvation energies $\delta E$ above the critical endpoints, Fig. 6(e)], the pore is empty at no applied voltage, and counterions only enter the pore when the applied potential matches the unfavorable resolvation energy; hence, there is a peak in capacitance when charging starts at $e V \approx \delta E$ [Fig. 6(a)]. Subsequently, a separate regime of packing like charges starts and persists 

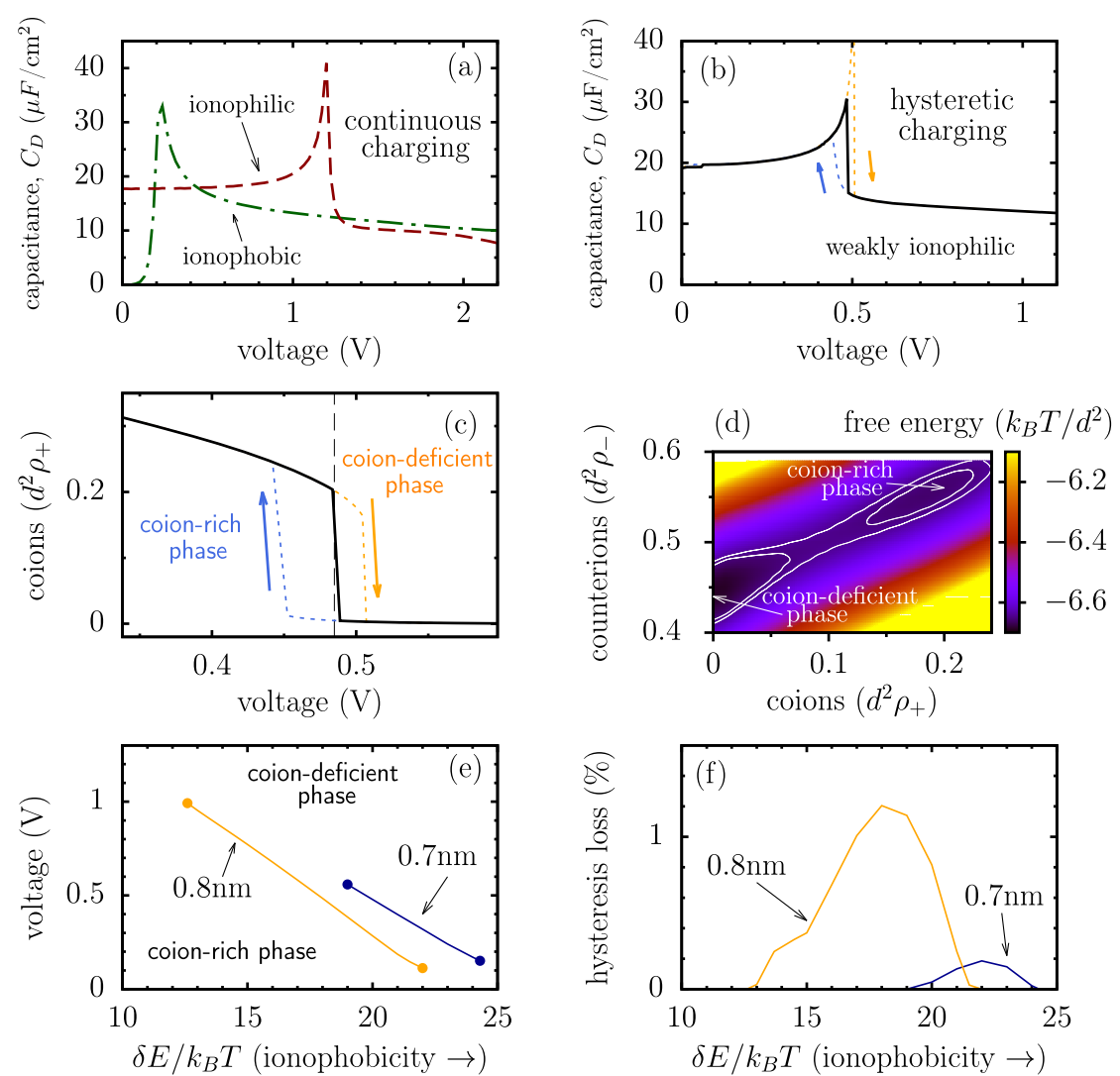

FIG. 6. Charging proceeds via a discontinuous phase transition for some parameter regimes. In all plots, ion diameter $d=0.5 \mathrm{~nm}$, and the mean-field theory is used to compute the thermodynamic properties. (a) Capacitance as a function of voltage in the continuous charging regime. The ionophilic and ionophobic pores have resolvation energies $\delta E=10 k_{B} T$ and $\delta E=32 k_{B} T$, respectively, and width $L=0.8 \mathrm{~nm}$. (b) Capacitance as a function of voltage in the discontinuous charging regime. The solid curve indicates the minimum freeenergy path, and the dotted curves denote the hysteresis loop with the arrows showing charging or discharging routes. The curve is plotted for $\delta E=18 k_{B} T$ and $L=0.8 \mathrm{~nm}$. (c) Discontinuous charging is connected with an abrupt expulsion of coions. We identify here coion-deficient and coion-rich phases with vanishing and appreciable amounts of coions in the pore, respectively. The arrows show charging and discharging paths, which follow the metastable branches denoted by dotted lines, with the solid curve giving the minimum energy path, as in panel (b). The vertical dashed line marks a voltage $V_{\text {coex }} \approx 0.49$ volts at which the two phases have the same free energy and coexist. (d) The free-energy landscape for applied voltage $V_{\text {coex }}$ corresponding to the dashed line in panel (c). White lines are a contour plot of constant free energy, and the arrows point to the ion densities that simultaneously minimize the free energy at coexistence. The contour plot has been obtained by evaluating the free energy for given densities of cations and anions using Eq. (3). (e) The phase diagram plotted for different slit widths. (f) The percentage of energy that is lost because of hysteresis during discharging of a nanopore charged at 1.3 volts (see Appendix A 4).

until the applied potential surpasses the interaction energy between coions. Beyond that point, the capacitance falls off rapidly.

The situation is different for strongly ionophilic pores with $\delta E$ below the critical endpoints, where the pore is nearly fully occupied with ions at zero voltage. At low voltages, charging proceeds via swapping of coions for counterions and coion desorption, while at higher voltages (above $\approx 1.2 \mathrm{~V}$ ), it is the counterion adsorption that drives the charging. It has been shown elsewhere [48] that adsorption leads to low capacitance, as compared to swapping and desorption, and hence the drop in the differential capacitance $C_{D}(V)$ at $V \approx 1.2 \mathrm{~V}$ [see the dashed line in Fig. 6(a)].
For ionophilicities between the two critical endpoints, charging proceeds via a large discontinuous drop in capacitance and ion densities [Figs. 6(b) and 6(c), respectively]. At low voltages, charging is driven by swapping of coions for counterions and coion expulsion, but at the point of the phase transition, the system expels all coions. This is similar to strongly ionophilic pores [Fig. 6(a)], except that this process is discontinuous, and we can observe two coexisting phases [14]: the coion-deficient phase, with a vanishing number of coions in the pore, and the coion-rich phase, with essentially nonzero coion density [Figs. 6(c) and 6(d)]. Within a small voltage window around this coexistence point, one of the two phases is metastable, and there is a free-energy barrier between them [Fig. 6(d)]; as 
we will see, this may have important implications for energy storage. Beyond the metastability window, charging occurs solely via counterion adsorption.

This voltage-induced abrupt expulsion of coions and the drop in the total ion density have recently been observed in atomistic molecular dynamics simulations of [C2mim] [FSI] and [C4mim][TFSI] ionic liquids in (0.75-nm-wide) slit nanopores [35]. However, in these studies, the drop in coion density is smoother than we observe, which is most likely due to finite-size effects. Indeed, in our theory, the pore is formally infinite in the lateral directions, while in the simulations of Ref. [35], the pore is only about a few tens of nanometers long.

Our results are also in line with recent NMR experiments $[44,52]$, which show that charging proceeds continuously (i.e. without phase transitions or accompanying abrupt coion expulsion) for $\left[\mathrm{PEt}_{4}\right]\left[\mathrm{BF}_{4}\right]$ ionic liquids and $\mathrm{KOH}$-activated carbon pores, which are known to be strongly ionophilic [8]. While it is difficult to calculate the resolvation energy (ionophobicity) for this system, Fig. 6(e) suggests that it must at least be lower than $12 k_{B} T$. This observation is consistent with our estimate of the crossover between the ionophilic and ionophobic pores which occurs at $\delta E_{\text {crossover }} \approx 17 k_{B} T$ for the 1 -nmwide pore of Ref. [44] (see Sec. II).

Figure 6(e) shows that the transition voltage increases with decreasing ionophobicity, as both counterions and coions are favorably adsorbed into the pore, making it more difficult to expel the coions. The window of ionophilicities for which a phase transition occurs is wider for larger pores, where electrostatic interactions are stronger and more long-ranged.

A signature of a discontinuous phase transition is hysteresis as the system may follow the metastable (locally stable) branch. The dotted lines in Figs. 6(b) and 6(c) show that the branch followed by the system when the voltage is increased is different from that followed by the system for decreasing voltage. Figure 6(f) shows that a significant amount of energy is lost per cycle as a consequence of hysteresis, with wider pores producing larger energy losses. In practical applications, the regime where charging proceeds via a first-order phase transition should therefore be avoided, but this hysteresis loop can be used to probe the properties of the supercapacitor system experimentally. However, it is likely that these transitions will be smoothed out by the distribution of pore sizes [53] in typically used porous electrodes (for instance, in carbide-derived carbons [3]), making it difficult to capture them directly by in situ NMR spectroscopy $[54,55]$ or in electrochemical quartz crystal microbalance experiments [56-58]. However, novel graphene-based nanoporous electrodes [6,7], which consist of nearly unimodal well-aligned slit pores with controllable pore widths, seem a promising candidate for validating our predictions experimentally. Although the quantum capacitance of these materials, which we have neglected in this work, may change the values of the total capacitance [5961], we do not expect that it will affect the existence of phase transitions or their order, as they result solely from the competition between entropic effects (due to confinement) and screened interionic interactions induced by the conducting pore walls. Nevertheless, it would be interesting to analyze the role that the quantum capacitance may play in energy storage and hysteretic charging.

\section{CONCLUSION}

Using a mean-field model for charge storage in 2D nanoconfinement and Monte Carlo simulations of the corresponding system in $3 \mathrm{D}$, we have demonstrated the possibility of simultaneously boosting capacitance, accelerating charging, and avoiding hysteresis in nanoporous supercapacitors. On the one hand, our calculations show that high capacitance can be achieved for electrolytes with small ions and close-fitting pores [inset of Fig. 5(a)]. On the other hand, we find that the long-espoused paradigm that equates narrow pores with necessarily larger capacitance does not always hold: Entropic effects may produce a second, more-pronounced peak in capacitance for relatively wide ionophobic pores and room-temperature ionic liquids [Figs. 3(a) and 4(b)]. Similarly, there exists an optimal ion diameter when the ratio between the ion diameter $(d)$ and the pore width $(L)$ is kept constant, suggesting an optimization of supercapacitors with respect to the $(d, L)$ pair rather than the pore size alone (Fig. 5).

For nonzero applied voltages, a voltage-induced discontinuous phase transition is predicted by the model, and the phase diagram has two critical endpoints corresponding to the limit of very ionophobic and ionophilic pores (Fig. 6). The phase transition gives rise to hysteresis and a sizable energy loss, but can crucially be avoided by either reducing the operating voltage of the capacitor or by pushing the pore ionophilicity away from the critical endpoints (for instance, by making pores more ionophobic). Thus, the capacitance-power-hysteresis trilemma can be resolved by judiciously tuning material parameters and operating ranges.

Ion diameter and pore width are relatively straightforward to tune experimentally; our framework shows how to optimize both parameters together. We also single out a key outstanding challenge for further experimentation-the controlled tuning of the ionophobicity or ionophilicity of the pore. The ionophilicity can be controlled by changing the ion-pore nonelectrostatic interaction. Consider a system where, in addition to ion-pore van der Waals interactions, the ion has multiple conformational states with different effective diameters (for example, expanded versus folded alkyl chains on an ionic liquid ion). If the pore separation in this system is less than the effective diameter of the lowestenergy conformation, then entering the pore will require the ion to adopt a higher conformation energy and thus incur a conformational energy penalty. Similarly, if the pore walls 
are flexible and ions can only enter by deforming the walls (as observed in Refs. [62,63]), the elastic energy will result in an unfavorable ion-pore nonelectrostatic interaction. This interaction could also be controlled by the addition of surfactants $[64,65]$ or solvents [44-46], or by using ionic liquid mixtures [66], or via functionalization of porous carbons [8]. However, experimental studies of ionophilicity are currently scarce, and we hope that our theory will provide a framework to assess and direct future efforts to address this.

The data points used for each figure can be accessed in Ref. [74].

\section{ACKNOWLEDGMENTS}

This work is supported by a EPSRC Doctoral Training Award and Fulbright Scholarship to A. A. L. S. K. acknowledges COST Action MP1004 for supporting his short-time scientific visit to Imperial College London where part of this work was completed, and appreciates fruitful discussions with Alexei Kornyshev (Imperial College).

\section{APPENDIX A: IMPROVED MEAN-FIELD APPROXIMATION}

The Helmholtz free energy $F\left(\rho_{+}, \rho_{-}\right)$per unit area of a homogeneous ionic liquid in a narrow-slit nanopore is given by

$$
\beta F=U_{\mathrm{el}}\left(\rho_{+}, \rho_{-}\right)-S\left(\rho_{+}, \rho_{-}\right)+\sum_{\alpha= \pm} h_{\alpha} \rho_{\alpha},
$$

where $\beta=\left(k_{B} T\right)^{-1}\left(k_{B}\right.$ is the Boltzmann constant and $T$ the temperature), $\rho_{ \pm}$are the two-dimensional ion densities, and it is convenient to introduce $\rho=\rho_{+}+\rho_{-}$and $c=\rho_{+}-\rho_{-}$. Here, $k_{B} T U_{\mathrm{el}}$ is the contribution to free energy due to electrostatic interactions, $k_{B} T S$ is the excluded volume entropic contribution, and $k_{B} T h_{\alpha}$ is the electrochemical potential of the ion species (see below).

\section{Electrostatic interactions}

The electrostatic interaction can be expressed in terms of direct correlation functions $\mathcal{C}_{\alpha \beta}\left(x, x^{\prime}\right)$ using a functional Taylor expansion

$$
U_{\mathrm{el}}\left(\rho_{+}, \rho_{-}\right) \approx \sum_{\alpha, \beta= \pm} \int \mathrm{d} x \int \mathrm{d} x^{\prime} \mathcal{C}_{\alpha \beta}\left(x, x^{\prime}\right) \rho_{\alpha} \rho_{\beta},
$$

with $\mathcal{C}_{\alpha \beta}\left(x, x^{\prime}\right)$ computed using the Ornstein-Zernicke relation with an appropriate closure [67]. However, here we make a simplifying assumption in the spirit of the random phase approximation (RPA). In RPA, the direct correlation function is approximated by the interaction potential, which is valid for asymptotically weak interactions but overestimates the correlations at close particle separations [67]. To improve on this, we assume that the direct correlation function is essentially negligible for distances less than the average particle separation; beyond this distance, the interaction is weak and can be approximated by the classical RPA. As such, $\mathcal{C}_{\alpha \beta}\left(x, x^{\prime}\right) \approx$ $v_{\alpha \beta}\left(x-x^{\prime}\right) \theta\left(\left|x-x^{\prime}\right|-R_{\mathrm{c}}\right)$, where $v_{\alpha \beta}\left(x-x^{\prime}\right)$ is the electrostatic interaction kernel and $R_{\mathrm{c}}=1 / \sqrt{\pi \rho}$ is the average separation between particles (this result is also known as the cutout disc approximation; see Ref. [68]).

The interaction potential between two point charges confined in a slit metallic nanopore and separated by distance $r$ is given by [69]

$$
\begin{aligned}
v_{\alpha \beta}\left(r, z_{1}, z_{2}\right)= & \frac{4 q_{\alpha} q_{\beta}}{\varepsilon_{p} L} \sum_{n=1}^{\infty} K_{0}(\pi n r / L) \sin \left(\pi n z_{1} / L\right) \\
& \times \sin \left(\pi n z_{2} / L\right),
\end{aligned}
$$

where $q_{\alpha}$ and $q_{\beta}$ are charges, $z_{1}$ and $z_{2}$ are ion positions across the pore (within the mean-field model, we assume $z_{1,2}=L / 2$, but we use the full potential in our Monte Carlo simulations, see below), and $\varepsilon_{p}$ is the dielectric constant in the pore; we have taken $\varepsilon_{p}=2$ in all calculations, but we note that $\varepsilon_{p}$, in principle, depends on the pore width (and voltage), and this dependence may have a profound effect on the system's behavior [70].

For monovalent ions, we obtain [14]

$U_{\mathrm{el}}(c, \rho)=4 c^{2} R_{c}(\rho) l_{B} \sum_{n=1}^{\infty} \frac{\sin ^{2}(\pi n / 2)}{n} K_{1}\left(\pi n R_{c}(\rho) / L\right)$,

where $l_{B}=e^{2} /\left(\varepsilon_{p} k_{B} T\right)$ is the thermal Bjerrum length. It is easy to see that at constant $L / d$, the functional dependence of the potential $v_{\alpha \beta}$ on $d$ is the same as on $L$ at constant $d$.

\section{Entropic contributions}

The entropic contribution in Eq. (A1) is modeled here based on the analytically solvable 2D scaled particle theory. The typical pore separation is only slightly larger than the ion diameter, and thus, we can take into account the positional disorder of ions normal to the pore surface as a perturbation to the otherwise 2D system - this effectively renormalizes the ion diameter. Thus, the entropy is given as a sum of the ideal gas contribution and hard core exclusion,

$S\left(\rho_{+}, \rho_{-}\right)=\sum_{\alpha= \pm} \tilde{\rho}_{\alpha} \ln \tilde{\rho}_{\alpha}+\tilde{\rho}\left[\frac{\tilde{\eta}^{2}}{1-\tilde{\eta}}-\ln (1-\tilde{\eta})\right]$,

where $\tilde{\eta}(\rho)=\pi \rho \sigma^{2} / 4=\pi \tilde{\rho} d^{2} / 4$ is the effective ion packing fraction and $\sigma(d, L, \rho)$ is a renormalized diameter that accounts for the out-of-plane packing of ions. Schmidt and Löwen [71,72] showed that 


$$
\sigma^{2}(d, L, \rho)=d^{2}+\frac{1}{\alpha(\rho)}-\frac{L \exp \left(\alpha(\rho) L^{2} / 4\right)}{\sqrt{\pi \alpha(\rho)} \operatorname{erfi}(\sqrt{\alpha(\rho)} L / 2)},
$$

where $\operatorname{erfi}(z)$ is the imaginary error function, and

$$
\alpha(\rho)=\pi \rho g(\rho)=\pi \rho \frac{1-\tilde{\eta}(\rho) / 2}{(1-\tilde{\eta}(\rho))^{2}}
$$

is the average density at contact and $g(\rho)$ the $2 \mathrm{D}$ pair correlation function evaluated at contact.

\section{Electrochemical potential}

The electrochemical potential (in infinite dilution) is given by

$h_{ \pm}= \pm u+\delta E_{\mathrm{self}}+\delta E_{ \pm}= \pm u-\frac{l_{B}}{L} \ln 2+\delta E_{ \pm}$.

Here, the first term results from the applied voltage, and the second term originates from the ion self-energy

$$
\begin{aligned}
\delta E_{\text {self }}(z) & =\lim _{r \rightarrow 0}\left(\phi(r)-\frac{1}{r}\right) \\
& =\frac{l_{B}}{L} \int_{0}^{\infty}\left[\frac{\sinh (Q(1-z / L)) \sinh (Q z / L)}{\sinh (Q)}-\frac{1}{2}\right] d Q,
\end{aligned}
$$

where $z$ is the position across the pore and $r$ the distance to the charge (see Ref. [14]). At the pore mid-plane $z=L / 2$, which gives $\delta E_{\text {self }}(L / 2)=-l_{B} \ln (2) / L$. Again, $\delta E_{\text {self }}$ depends on $L$ in the same way as on $d$ at constant $L / d$. The last term in Eq. (A8), $\delta E_{ \pm}$, is the "resolvation energy," which is the energy of transferring an ion from the bulk of a supercapacitor into the pore in the absence of other ions.

\section{Stored energy and hysteretic energy loss}

The energy (per surface area) stored in a nanopore by charging it from $u=u_{1}$ to $u=u_{2}$ is

$$
\mathcal{E}\left(u_{1}, u_{2}\right)=\int_{u_{1}}^{u_{2}} u C_{D}(u) \mathrm{d} u,
$$

where $C_{D}(u)=\mathrm{d} Q / \mathrm{d} V$ is differential surface-specific capacitance. To obtain an energy lost due to hysteresis [Fig. 6(f)], we first calculated the energy stored when charging a nanopore from $u_{1}=0$ to $u_{2}=V=1.3 \mathrm{~V}$ along the charging path [orange lines and arrows in Fig. 6(b)], $\mathcal{E}_{\text {charg }}=\mathcal{E}(0, V)$; and then the energy released by fully discharging it along the discharging path [blue lines and arrows in Fig. 6(b)], $\mathcal{E}_{\text {discharg }}=-\mathcal{E}(V, 0)$. The hysteretic energy loss is shown as a percentage of the stored energy lost in hysteresis, i.e., $\left(\mathcal{E}_{\text {charg }}-\mathcal{E}_{\text {discharg }}\right) / \mathcal{E}_{\text {charg }}$.

\section{APPENDIX B: GRAND CANONICAL MONTE CARLO SIMULATIONS}

We used the same method as in Ref. [36], so we only summarize the simulation method here and refer the reader to Ref. [36] for further details.

Ionic liquid molecules are modeled as charged hard spheres, but instead of the Coulomb potential, we use the analytical solution (A3) for the ion-ion interactions. Ionpore wall interactions are captured by potential (A9), which accounts for image forces. The resolvation energy $(\delta E)$ and the applied voltage $(V)$ are subsumed into the chemical potential in the grand canonical simulations, $\mu_{ \pm}^{\text {(sim) }}=$ $\delta E \pm e V$, where $e$ is the elementary charge, and $\delta E$ is the resolvation energy, as before. In all our simulations, we took the temperature $T=328 \mathrm{~K}$ and the relative dielectric constant inside pores $\varepsilon_{p}=2$.

To calculate differential capacitance, we numerically differentiated the accumulated charge obtained from Monte Carlo simulations with respect to voltage using the Holoborodko method with seven points [73].

[1] M. Lu, F. Beguin, and E. Frackowiak, Supercapacitors: Materials, Systems and Applications (Wiley-VCH, Verlag, 2013).

[2] P. Simon and Y. Gogotsi, Capacitive Energy Storage in Nanostructured Carbon-Electrolyte Systems, Acc. Chem. Res. 46, 1094 (2013).

[3] P. Simon and Y. Gogotsi, Materials for Electrochemical Capacitors, Nat. Mater. 7, 845 (2008).

[4] E. Frackowiak, Carbon Materials for Supercapacitor Application, Phys. Chem. Chem. Phys. 9, 1774 (2007).

[5] Y. Gogotsi, A. Nikitin, H. Ye, W. Zhou, J. E. Fischer, B. Yi, H. C. Foley, and M. W. Barsoum, Nanoporous CarbideDerived Carbon with Tunable Pore Size, Nat. Mater. 2, 591 (2003).

[6] J. J. Yoo, K. Balakrishnan, J. S. Huang, V. Meunier, B. G. Sumpter, A. Srivastava, M. Conway, A. L. M. Reddy, J. Yu, R. Vajtai, and P. M. Ajayan, Ultrathin Planar Graphene Supercapacitors, Nano Lett. 11, 1423 (2011).

[7] X. Yang, C. Cheng, Y. Wang, L. Qiu, and D. Li, LiquidMediated Dense Integration of Graphene Materials for Compact Capacitive Energy Storage, Science 341, 534 (2013).

[8] F. W. Richey, C. Tran, V. Kalra, and Y. A. Elabd, Ionic Liquid Dynamics in Nanoporous Carbon Nanofibers in Supercapacitors Measured with in Operando Infrared Spectroelectrochemistry, J. Phys. Chem. C 118, 21846 (2014).

[9] B. E. Conway, Electrochemical Supercapacitors: Scientific Fundamentals and Technological Applications (Springer, Berlin, 1999).

[10] E. Raymundo-Piñero, K. Kierczek, J. Machnikowski, and F. Béguin, Relationship between the Nanoporous Texture of Activated Carbons and Their Capacitance Properties in Different Electrolytes, Carbon 44, 2498 (2006).

[11] J. Chmiola, G. Yushin, Y. Gogotsi, C. Portet, P. Simon, and P. L. Taberna, Anomalous Increase in Carbon Capacitance 
at Pore Sizes Less Than 1 Nanometer, Science 313, 1760 (2006).

[12] C. Largeot, C. Portet, J. Chmiola, P. L. Taberna, Y. Gogotsi, and P. Simon, Relation between the Ion Size and Pore Size for an Electric Double-Layer Capacitor, J. Am. Chem. Soc. 130, 2730 (2008).

[13] R. Lin, P. Huang, J. Segalini, C. Largeot, P.-L. Taberna, J. Chmiola, Y. Gogotsi, and P. Simon, Solvent Effect on the Ion Adsorption from Ionic Liquid Electrolyte into SubNanometer Carbon Pores, Electrochim. Acta 54, 7025 (2009).

[14] S. Kondrat and A. Kornyshev, Superionic State in DoubleLayer Capacitors with Nanoporous Electrodes, J. Phys. Condens. Matter 23, 022201 (2011).

[15] C. C. Rochester, A. A. Lee, G. Pruessner, and A. A. Kornyshev, Interionic Interactions in Electronically Conducting Confinement, ChemPhysChem 14, 4121 (2013).

[16] A. Goduljan, F. Juarez, L. Mohammadzadeh, P. Quaino, E. Santos, and W. Schmickler, Screening of Ions in Carbon and Gold Nanotubes-A Theoretical Study, Electrochem. Comm. 45, 48 (2014).

[17] L. Mohammadzadeh, A. Goduljan, F. Juarez, P. Quaino, E. Santos, and W. Schmickler, Nanotubes for Charge Storage-Towards an Atomistic Model, Electrochim. Acta 162, 11 (2015).

[18] A. A. Lee, D. Vella, and A. Goriely, Quantum Capacitance Modifies Interionic Interactions in Semiconducting Nanopores, Europhys. Lett. 113, 38005 (2016).

[19] C. Merlet, B. Rotenberg, P. A. Madden, P.-L. Taberna, P. Simon, Y. Gogotsi, and M. Salanne, On the Molecular Origin of Supercapacitance in Nanoporous Carbon Electrodes, Nat. Mater. 11, 306 (2012).

[20] C. Merlet, C. Péan, B. Rotenberg, P. A. Madden, B. Daffos, P. L. Taberna, P. Simon, and M. Salanne, Highly Confined Ions Store Charge More Efficiently in Supercapacitors, Nat. Commun. 4, 2701 (2013).

[21] P. Wu, J. Huang, V. Meunier, B. G. Sumpter, and R. Qiao, Complex Capacitance Scaling in Ionic Liquids-Filled Nanopores, ACS Nano 5, 9044 (2011).

[22] R. Qiao, V. Meunier, and B. G. Sumpter, Voltage Dependent Charge Storage Modes and Capacity in Subnanometer Pores, J. Phys. Chem. Lett. 3, 1732 (2012).

[23] J. Vatamanu, Z. Hu, D. Bedrov, C. Perez, and Y. Gogotsi, Increasing Energy Storage in Electrochemical Capacitors with Ionic Liquid Electrolytes and Nanostructured Carbon Electrodes, J. Phys. Chem. Lett. 4, 2829 (2013).

[24] R. Mysyk, E. Raymundo-Pińero, and F. Béguin, Saturation of Subnanometer Pores in an Electric Double-Layer Capacitor, Electrochem. Comm. 11, 554 (2009).

[25] S. Kondrat and A. Kornyshev, Charging Dynamics and Optimization of Nano-Porous Supercapacitors, J. Phys. Chem. C 117, 12399 (2013).

[26] A. A. Lee, S. Kondrat, G. Oshanin, and A. A. Kornyshev, Charging Dynamics of Supercapacitors with Narrow Cylindrical Nanopores, Nanotechnology 25, 315401 (2014).

[27] S. Kondrat, P. Wu, R. Qiao, and A. A. Kornyshev, Accelerating Charging Dynamics in Subnanometre Pores, Nat. Mater. 13, 387 (2014).

[28] V. Lockett, R. Sedev, J. Ralston, M. Horne, and T. Rodopoulos, Differential Capacitance of the Electrical
Double Layer in Imidazolium-Based Ionic Liquids: Influence of Potential, Cation Size, and Temperature, J. Phys. Chem. C 112, 7486 (2008).

[29] W. Zhou, S. Inoue, T. Iwahashi, K. Kanai, K. Seki, T. Miyamae, D. Kim, Y. Katayama, and Y. Ouchi, Double Layer Structure and Adsorption/Desorption Hysteresis of Neat Ionic Liquid on Pt Electrode Surface-An In-Situ IRVisible Sum-Frequency Generation Spectroscopic Study, Electrochem. Comm. 12, 672 (2010).

[30] A. Uysal, H. Zhou, G. Feng, S. S. Lee, S. Li, P. Fenter, P. T. Cummings, P. F. Fulvio, S. Dai, J. K. McDonough, and Y. Gogotsi, Structural Origins of Potential Dependent Hysteresis at the Electrified Graphene/Ionic Liquid Interface, J. Phys. Chem. C 118, 569 (2014).

[31] B. Rotenberg and M. Salanne, Structural Transitions at Ionic Liquid Interfaces, J. Phys. Chem. Lett. 6, 4978 (2015).

[32] M. Drüschler, B. Huber, S. Passerini, and B. Roling, Hysteresis Effects in the Potential-Dependent Double Layer Capacitance of Room Temperature Ionic Liquids at a Polycrystalline Platinum Interface, J. Phys. Chem. C 114, 3614 (2010).

[33] K. Kiyohara, T. Sugino, and K. Asaka, Phase Transition in Porous Electrodes, J. Chem. Phys. 134, 154710 (2011).

[34] L. Xing, J. Vatamanu, O. Borodin, and D. Bedrov, On the Atomistic Nature of Capacitance Enhancement Generated by Ionic Liquid Electrolyte Confined in Subnanometer Pores, J. Phys. Chem. Lett. 4, 132 (2013).

[35] J. Vatamanu, M. Vatamanu, and D. Bedrov, Non-Faradic Energy Storage by Room Temperature Ionic Liquids in Nanoporous Electrodes, ACS Nano 9, 5999 (2015).

[36] S. Kondrat, N. Georgi, M. V. Fedorov, and A. A. Kornyshev, A Superionic State in Nano-Porous Double-Layer Capacitors: Insights from Monte Carlo Simulations, Phys. Chem. Chem. Phys. 13, 11359 (2011).

[37] K. Kiyohara, H. Shioyama, T. Sugino, and K. Asaka, Phase Transition in Porous Electrodes. II. Effect of Asymmetry in the Ion Size, J. Chem. Phys. 136, 094701 (2012).

[38] K. Kiyohara, H. Shioyama, T. Sugino, K. Asaka, Y. Soneda, K. Imoto, and M. Kodama, Phase Transition in Porous Electrodes. III. For the Case of a Two Component Electrolyte, J. Chem. Phys. 138, 234704 (2013).

[39] D. Jiang, Z. Jin, and J. Wu, Oscillation of Capacitance inside Nanopores, Nano Lett. 11, 5373 (2011).

[40] D. Jiang and J. Wu, Unusual Effects of Solvent Polarity on Capacitance for Organic Electrolytes in a Nanoporous Electrode, Nanoscale 6, 5545 (2014).

[41] J. F. Jover, R. Lugo, H. Toulhoat, P. Simon, and T. De Bruin, Screening Methodology for the Efficient Pairing of Ionic Liquids and Carbonaceous Electrodes Applied to Electric Energy Storage, J. Phys. Chem. C 118, 864 (2014).

[42] From Ref. [41], the energy to transfer an ion pair from the bulk to vacuum is approximately to $50-85 k_{B} T$, and the energy to dissociate an ion pair at vacuum is approximately to $80-140 k_{B} T$; therefore, the desolvation energy of a single ion is approximately to $65-115 k_{B} T$.

[43] This is the estimate obtained from Ref. [41], where a cylindrical geometry is studied. Our estimate is obtained by taking the large radius asymptotic value of the ion-wall van der Waals interaction energy, i.e., $E_{\mathrm{vdw}, \mathrm{slit}} \approx$ $2 E_{\mathrm{vdw}, \text { cylinder }}(R \rightarrow \infty)$, as the $R \rightarrow \infty$ asymptotic value of 
the van der Waals interaction energy corresponds to the interaction energy between an ion and one side of the pore.

[44] J. M. Griffin, A. C. Forse, W.-Y. Tsai, P.-L. Taberna, P. Simon, and C. P. Grey, In Situ NMR and Electrochemical Quartz Crystal Microbalance Techniques Reveal the Structure of the Electrical Double Layer in Supercapacitors, Nat. Mater. 14, 812 (2015).

[45] A. C. Forse, J. M. Griffin, C. Merlet, P. M. Bayley, H. Wang, P. Simon, and C. P. Grey, NMR Study of Ion Dynamics and Charge Storage in Ionic Liquid Supercapacitors, J. Am. Chem. Soc. 137, 7231 (2015).

[46] C. C. Rochester, S. Kondrat, G. Pruessner, and A. A. Kornyshev, Charging Ultra-Nanoporous Electrodes with Size-Asymmetric Ions Assisted by Apolar Solvent, doi: 10.1021/acs.jpcc.5b12730.

[47] A. A. Lee, S. Kondrat, and A. A. Kornyshev, Single-File Charge Storage in Conducting Nanopores, Phys. Rev. Lett. 113, 048701 (2014).

[48] S. Kondrat and A. A. Kornyshev, Pressing a Spring: What Does It Take to Maximize the Energy Storage in Nanoporous Supercapacitors?, Nanoscale Horiz. 1, 45 (2016).

[49] G. Feng and P. T. Cummings, Supercapacitor Capacitance Exhibits Oscillatory Behavior as a Function of Nanopore Size, J. Phys. Chem. Lett. 2, 2859 (2011).

[50] O. Pizio, S. Sokolowski, and Z. Sokolowska, Electric Double Layer Capacitance of Restricted Primitive Model for an Ionic Fluid in Slit-Like Nanopores: A Density Functional Approach, J. Chem. Phys. 137, 234705 (2012).

[51] We note, however, that this reasoning is valid for porewidth-independent (or only weakly dependent) resolvation energies. Although this is not generally the case, it seems reasonable to expect it to hold for small variations of the slit width $d<L<2 d$ considered in this work.

[52] J. M. Griffin, A. C. Forse, H. Wang, N. M. Trease, P.-L. Taberna, P. Simon, and C. P. Grey, Ion Counting in Supercapacitor Electrodes Using NMR Spectroscopy Faraday Discuss. 176, 49 (2014).

[53] S. Kondrat, C. R. Pérez, V. Presser, Y. Gogotsi, and A. A. Kornyshev, Effect of Pore Size and Its Dispersity on the Energy Storage in Nanoporous Supercapacitors, Energy Environ. Sci. 5, 6474 (2012).

[54] H. Wang, A. C. Forse, J. M. Griffin, N. M. Trease, L. Trognko, P.-L. Taberna, P. Simon, and C. P. Grey, In Situ NMR Spectroscopy of Supercapacitors: Insight into the Charge Storage Nechanism, J. Am. Chem. Soc. 135, 18968 (2013).

[55] A. C. Forse, J. M. Griffin, H. Wang, N. M. Trease, V. Presser, Y. Gogotsi, P. Simone, and C. P. Grey, Nuclear Magnetic Resonance Study of Ion Adsorption on Microporous Carbide-Derived Carbon, Phys. Chem. Chem. Phys. 15, 7722 (2013).

[56] M. D. Levi, G. Salitraand Naomi Levy, D. Aurbach, and J. Maier, Application of a Quartz-Crystal Microbalance to Measure Ionic Fluxes in Microporous Carbons for Energy Storage, Nat. Mater. 8, 872 (2009).
[57] M. D. Levi, S. Sigalov, D. Aurbach, and L. Daikhin, In Situ Electrochemical Quartz Crystal Admittance Methodology for Tracking Compositional and Mechanical Changes in Porous Carbon Electrodes, J. Phys. Chem. C 117, 4876 (2013).

[58] W.-Y. Tsai, P.-L. Taberna, and P. Simon, Electrochemical Quartz Crystal Microbalance (EQCM) Study of Ion Dynamics in Nanoporous Carbons, J. Am. Chem. Soc. 136, 8722 (2014).

[59] J. Xia, F. Chen, J. Li, and N. Tao, Measurement of the Quantum Capacitance of Graphene, Nat. Nanotechnol. 4, 505 (2009).

[60] M. D. Stoller, C. W. Magnuson, Y. Zhu, S. Murali, J. Won Suk, R. Piner, and R. S. Ruoff, Interfacial Capacitance of Single Layer Graphene, Energy Environ. Sci. 4, 4685 (2011).

[61] A. A. Kornyshev, N. B. Luque, and W. Schmickler, Differential Capacitance of Ionic Liquid Interface with Graphite: The Story of Two Double Layers, J. Solid State Electrochem. 18, 1345 (2014).

[62] F. Kaasik, T. Tamm, M. M. Hantel, E. Perre, A. Aabloo, E. Lust, M. Z. Bazant, and V. Presser, Anisometric Charge Dependent Swelling of Porous Carbon in an Ionic Liquid, Electrochem. Comm. 34, 196 (2013).

[63] M. M. Hantel, D. Weingarth, and R. Kötz, Parameters Determining Dimensional Changes of Porous Carbons During Capacitive Charging, Carbon 69, 275 (2014).

[64] K. Fic, G. Lot, and E. Frackowiak, Electrochemical Properties of Supercapacitors Operating in Aqueous Electrolyte with Surfactants, Electrochim. Acta 55, 7484 (2010).

[65] K. Fic, G. Lot, and E. Frackowiak, Effect of Surfactants on Capacitance Properties of Carbon Electrodes, Electrochim. Acta 60, 206 (2012).

[66] R. Lin, P.-L. Taberna, S. Fantini, V. Presser, C. R. Pérez, F. Malbosc, N. L. Rupesinghe, K. B. K. Teo, Y. Gogotsi, and P. Simon, Capacitive Energy Storage from -50 to $100{ }^{\circ} \mathrm{C}$ Using an Ionic Liquid Electrolyte, J. Phys. Chem. Lett. 2, 2396 (2011).

[67] J.-P. Hansen and J. R. MacDonald, Theory of Simple Liquids, 4th ed. (Elsevier, New York, 2014).

[68] A. A. Kornyshev and W. Schmickler, On the Coverage Dependence of the Partial Charge Transfer Coefficient, J. Electroanal. Chem. 202, 1 (1986).

[69] W. R. Smythe, Static and Dynamic Electricity (McGrawHill, New York, 1939).

[70] S. Kondrat, A. Kornyshev, F. Stoeckli, and T. A. Centeno, The Effect of Dielectric Constant on Capacitance of Nanoporous Electrodes, Electrochem. Comm. 34, 348 (2013).

[71] M. Schmidt and H. Löwen, Freezing between Two and Three Dimensions, Phys. Rev. Lett. 76, 4552 (1996).

[72] M. Schmidt and H. Löwen, Phase Diagram of Hard Spheres Confined between Two Parallel Plates, Phys. Rev. E 55, 7228 (1997).

[73] P. Holoborodko, Smooth Noise Robust Differentiators, http://www.holoborodko.com/pavel/numerical-methods/ numerical-derivative/smooth-low-noise-differentiators.

[74] DOI: 10.5287/bodleian:JNn1NqvD1. 